\title{
ENTRE LA ACLIMATACIÓN A LA ALTURA, LA ANTROPOLOGÍA MÉDICA Y LA UTOPÍA CIVILIZATORIA. CARTOGRAFÍA DE LA EVOLUCIÓN DEL PENSAMIENTO DE CARLOS MONGE MEDRANO SOBRE EL PROCESO SALUD-ENFERMEDAD DE POBLACIONES ANDINAS
}

\author{
Juan P. Murillo1,a
}

\begin{abstract}
RESUMEN
El presente estudio tiene como objetivo describir cómo se van desarrollando las ideas de Carlos Monge sobre las enfermedades de altura y cómo estas se van desplegando en el marco de los debates sobre las condiciones de vida de las poblaciones indígenas en el periodo 1928-1963. Postulamos que la forma cómo se constituyó el paradigma propuesto por Monge, las tensiones producidas desde varias corrientes alternativas y la forma cómo se resolvieron dichas contradicciones fueron centrales, tanto para el desarrollo posterior de distintas disciplinas científicas, como para sus distintas expresiones institucionales en el Perú. Se describe la trayectoria evolutiva de las ideas de Monge sobre adaptación de las poblaciones indígenas a la altura y los debates surgidos con otros colectivos investigativos en torno al consumo de la hoja de coca. Se plantea un análisis de las limitaciones de una mirada biomédica de la salud de las poblaciones andinas que, progresivamente, va siendo influenciada por el cambiante escenario social y político peruano en el contexto de la guerra fría.
\end{abstract}

Palabras clave: Mal de altura, Antropología médica; Coca; Perú (fuente: DeCS BIREME).

\section{BETWEEN THE ACCLIMATIZATION TO HIGH ALTITUDE, THE MEDICAL ANTHROPOLOGY AND THE CIVILIZING UTOPIA. MAPPING OF THE EVOLUTION OF THOUGHT OF CARLOS MONGE MEDRANO ON THE HEALTH-ILLNESS PROCESS OF ANDEAN POPULATIONS}

\begin{abstract}
The main objective of this study is to describe how the ideas of Carlos Monge respect to high altitude sickness developed and how these were being deployed in the framework of the discussions on the living conditions of indigenous populations in the period 1928-1963. I postulate that the form how the Monge's paradigm was proposed, the tensions produced by various alternative movements and the way these contradictions were resolved were central, both for the subsequent development of different scientific disciplines and for their different institutional expressions in Peru. In addition, this article describes the evolutionary trajectory of Monge's ideas about the adaptation of indigenous populations to high altitude and the discussions that emerged with other research groups around the consumption of coca leaf. It also presents an analysis of the limitations of the biomedical view regarding the health of the Andean populations, a view that was progressively influenced by the changing social and political scenario in the context of the Cold War.
\end{abstract}

Key words: Altitude sickness; Anthropology, medical; Coca; Peru (source: MeSH NLM).

\section{INTRODUCCIÓN}

Carlos Monge Medrano fue, sin lugar a dudas, uno de los más importantes científicos peruanos del siglo XX. Su contribución al desarrollo de la investigación biomédica en el Perú y su carácter central en la construcción de una tradición científica nacional han sido extensamente estudiados en diversos trabajos ${ }^{(1,2)}$; sin embargo, existen pocas aproximaciones que analizan las contribuciones de Monge, en términos de Ludwig Fleck (3), un estilo de pensamiento, que configuró un colectivo de pensamiento que tuvo la hegemonía del escenario investigativo peruano durante la primera mitad del siglo XX. El descubrimiento por parte de Carlos Monge de

Facultad de Medicina, Universidad Nacional Mayor de San Marcos

Médico cirujano

Recibido: 16/05/2017 Aprobado: 07/06/2017 En línea: 28/06/2017

Citar como: Murillo JP. Entre la aclimatación a la altura, la antropología médica y la utopía civilizatoria. Cartografía de la evolución del pensamiento de Carlos Monge Medrano sobre el proceso salud-enfermedad de poblaciones andinas. Rev Peru Med Exp Salud Publica. 2017;34(2):280-6. doi: 10.17843/rpmesp.2017.342.2880 
la "enfermedad de los Andes" en 1928, dio paso a la construcción de una visión biomédica del hombre andino, elemento que fue central para el desarrollo de la investigación en medicina en el Perú en la primera mitad del siglo XX. Postulamos que la forma cómo se constituyó el paradigma propuesto por Monge, las tensiones producidas desde varias corrientes alternativas y la forma cómo se resolvieron dichas contradicciones fueron centrales tanto, para el desarrollo posterior de distintas disciplinas científicas, como para sus distintas expresiones institucionales en el Perú. El objetivo de este artículo es describir cómo se van desarrollando las ideas de Monge y cómo estas se van desplegando en el marco de los debates sobre las condiciones de vida de las poblaciones indígenas en el periodo 1928-1963.

\section{CONSTRUYENDO UNA NUEVA IMAGEN EL HOMBRE ANDINO}

El conocimiento de la enfermedad de los Andes plantea un cambio radical en la forma de aproximarse a poblaciones como objetos de estudio, ya no ubicándolos como grupos disminuidos o degenerados por siglos de explotación y abandono, sino como una raza con enormes capacidades y potencialidades: Físicamente hemos hecho ver cuál es su capacidad para el trabajo, no igualada por raza alguna en esas zonas elevadas. A pesar de la minuciosidad de la descripción del habitante nativo de los andes, resalta un sentimiento de orgullo que, incluso Monge proyecta en primera persona cuando señala al describir sus funciones superiores Mentalmente, no es el caso hablar de nosotros mismos, pero bien pueden tomarse como información sus obras milenarias y el estado de floreciente estado espiritual del Cusco, para no citar, sino la cuna y capital del imperio de los incas ${ }^{(4)}$.

Como consecuencia del enorme éxito científico, Monge construye un relato que le permite situar sus hallazgos en el núcleo duro de la tradición médica peruana, planteando una línea de continuidad con el pensamiento de Hipólito Unanue, al establecer la centralidad del determinismo climático, para entender la vida y la enfermedad, de cómo el hombre andino es un producto de su medioambiente y que este determinismo climático constituye el corazón conceptual de la Escuela Médica Peruana ${ }^{(5)}$. Este elemento es un hito en el desarrollo del paradigma de determinismo climático propuesto por Monge, además, le añade la carga simbólica de unirlo a la tradición del pensamiento clásico de Unanue y, por lo tanto, le añade un sentido de identidad de un colectivo médico nacional.

Un año después, en la Segunda Conferencia Panamericana de Eugenesia y Homicultura realizada en Buenos Aires, en 1934, Monge expande la amplitud de su paradigma, al señalar que: Dentro de estas razas que pueblan el altiplano, hay un factor tan característico-geográfico- que hay derecho a establecer una antropogeografía del hombre de los Andes, completamente distinta a la antropogeografía incipiente y discutida que nosotros conocemos del hombre a nivel del mar. Posteriormente, plantea la influencia de la altura en la vida social, dada la activa movilidad poblacional originada por la necesidad de oxigenación, lo que influye en la dinámica de la vida productiva del país. Es en este escenario que lanza su concepto biología andina, al plantear la gran especificidad que tiene el hombre, como organismo vivo adaptado a la altitud $^{(6)}$. Al año siguiente, en otro evento internacional, construye una proyección histórica de "La agresión climática" a partir de las descripciones de los cronistas. Asocia los asentamientos de "mitimaes" a una política de ocupación del territorio, basada de la adaptación de poblaciones a diferentes pisos de altitud ${ }^{(7)}$.

El año 1941 es clave en la construcción teórica de la enfermedad de altura. En la lectura de su discurso al recibir el grado de Doctor Honoris Causa, de la Universidad de Chicago, donde luego de listar la gran cantidad de características biológicas señala: Las características biológicas mencionadas son distintas de las que corresponden a los hombres del nivel del mar. Por esta razón, los hombres de los Andes pueden ser considerados como pertenecientes a una variedad climatofisiológica de raza humana. En efecto, están enteramente relacionados con su ambiente geográfico: altura, radiación, humedad, ionización, etc. La conducta sociológica de tales hombres y el ambiente telúrico aparecen como un todo, como un sistema biológico indivisible, como unidad climatofisiológica. Tan significativo como la identificación de una variedad de raza humana, fue la detallada mención a las contribuciones de investigadores peruanos. En esta comunicación, que sería publicada en la revista Science en 1942, Monge cita a diez investigadores peruanos, lo cual es una muestra explícita de una activa comunidad científica local ${ }^{(8)}$.

El regreso de Monge de los Estados Unidos fue apoteósico. En una reunión de homenaje, donde participaron ministros de estado, el rector de la Universidad de San Marcos y gran parte de las instituciones médicas del país. El presidente de la Academia Nacional de Medicina, Dr. Juan Voto Bernales, señaló que el año 1928, con el descubrimiento de la enfermedad de los Andes se marcaba una etapa distinta en la metodología científica de la medicina peruana, sin embargo, destaca en Monge que: Se apartaba del tradicional sistema de producción personal y la apreciación unilateralizada. Comenzó a interesar al grupo, a buscar una colaboración que era una visión del problema trascendental, e inicia entonces la estructuración de una escuela, obra fundamental, cuyas producciones son bella y honrosa ejecutoria para la medicina peruana y augurales 
derroteros en la solución de problemas nacionales. Carlos Monge en su discurso de agradecimiento señaló: Sería perfectamente pretensioso suponer que un hombre pudiera atribuirse la obra ejecutada por la escuela médica peruana. Admito que fue posible y necesaria una idea directriz, y que la tuve ${ }^{(9)}$.

Sin embargo, el discurso de Monge fue más allá de un simple agradecimiento. Planteó en términos fundacionales lo que sería un programa de desarrollo colectivo de una nueva investigación, con una sólida ética base sustantiva del progreso que guía una investigación al servicio del desarrollo industrial del país, articulada a un dinámico relacionamiento con organizaciones internacionales y actores económicos del país. Terminó su discurso con un sentido compromiso: Ojalá que este homenaje que tanto conmueve, se traduzca en un movimiento arrollador al servicio de las razas autóctonas del altiplano ${ }^{(9)}$.

No obstante, a pesar del enorme consenso que logró alcanzar las ideas de Monge, ya a la mitad de la década del treinta, comenzaron a plantearse perspectivas distintas respecto a la problemática sanitaria del hombre andino. En un editorial de 1936 de la revista "Actualidad Médica Peruana", dirigida por Sergio Bernales, se proponía la necesidad de nuevas aproximaciones al estudio de las condiciones de vida y a los problemas de salud de las poblaciones indígenas. Proponían una mirada que entendían más integral al señalar: Aspiramos a una nueva forma del indigenismo, a una concepción que haya en el indio al ser, cuya evolución puede inducirse con criterios científicos...tal vez algunos juzguen excesiva esta pretensión, de encarar así una cuestión tan compleja y que hasta ahora se estimó como el campo propio de la sociología y la política. El pueblo, como entidad orgánica que es, debe ser considerado, tanto como criterio biológico, como histórico y sociológico ${ }^{(10)}$.

Desde Actualidad Médica Peruana se planteó una visión que se veía a sí misma como "complementaria" a la propuesta investigativa de la medicina de altura. La revista planteó una mirada del hombre andino desde la antropología física, con una cierta impronta eugenésica, como lo muestra la reproducción de trabajos de prestigiosos investigadores como José Imbelloni y Alejandro Lipschutz, además de la publicación de las investigaciones sobre biotipología del hombre andino de Carlos Gutiérrez Noriega ${ }^{(11,12)}$.

\section{VISIONES EN CONFLICTO: EL COMPLEJO DEBATE SOBRE LA COCA EN EL PERÚ}

Esta mirada del hombre andino comienza a ser cuestionada, de una manera directa, a partir de las investigaciones de Carlos Gutiérrez Noriega, desde la
Cátedra de Farmacología de la Facultad de Medicina de San Fernando y, posteriormente, desde el Departamento de Farmacología y Terapéutica Experimental del Instituto Nacional de Higiene y Salud Pública. Desde estas posiciones se comenzó a generar numerosa evidencia orientada al estudio de los mecanismos de acción de la cocaína, tanto a través de modelos de laboratorio en animales, como en observaciones experimentales en sujetos voluntarios. Gutiérrez Noriega partía de que el consumo habitual de la hoja de coca era una toxicomanía, que más que constituir un alimento adaptativo que facilita el proceso de aclimatación, el consumo fue un proceso histórico asociado al trabajo intensivo y a la explotación de los indígenas en el campo y en las minas. En términos generales, mostraba evidencias de un impacto potencial negativo para la salud y consideraba que el consumo de hojas de coca debería ser considerado uno de los más importantes problemas de Salud Pública, estimando entre alrededor de 1 millón de personas afectadas en el Perú ${ }^{(13)}$.

En este momento, la controversia estaba planteada y no solo desde un punto de vista académico. Las investigaciones de Gutiérrez Noriega recibían el apoyo del decano de la Facultad de Medicina, Sergio Bernales, quien impulsaba la creación de un nuevo instituto de investigación en el campo de la Farmacología y Terapéutica Experimental. Carlos Monge, primero dirige un informe al Decano, sobre sus actividades realizadas en 1946 en universidades norteamericanas, donde se reafirma en sus ideas sobre el hombre andino y remarca el consenso que tienen sus premisas entre sus pares de investigación en dichas instituciones (14). Posteriormente, publica en Anales de la Facultad, un ensayo que constituyó un ataque frontal, a las tesis de Gutiérrez Noriega. El artículo, titulado, "El problema de la coca en el Perú", comienza estableciendo un juicio de valor, no muy elegante que digamos, sobre las investigaciones críticas en torno a la coca, cuando afirma que: Mucho se ha escrito y muy poco se ha investigado sobre el coqueo en los altiplanos de América, para después colocar los diversos modelos y observaciones realizadas por Gutiérrez Noriega, en una categoría de ciencia extraordinaria, al afirmar que: Siempre tuvimos la impresión que para abordar este problema era absolutamente indispensable, en primer lugar, conocer la fisiología del hombre andino, cosa ignorada hasta los trabajos de la Escuela Médica Peruana y de los que han prescindido casi todos los investigadores que han tratado del coqueo. Posteriormente, parte de su premisa de que el hombre andino es fisiológicamente y químicamente diferente al hombre del nivel del mar, por lo que los efectos de la coca, señalados por las observaciones experimentales de Gutiérrez Noriega, no necesariamente deberían verificarse en la gran altitud. Posteriormente, apela a su /...experiencia clínica $y$ social para expresar sus dudas sobre la acción nociva 
de la coca, inclinándose más bien a admitir la necesidad e inocuidad de la droga en las condiciones extremas de la vida de altitud. En ese sentido, Monge remarca que: Es posible suponer que actúa como agente farmacológico potenciador de las relaciones humorales que permiten un mejor rendimiento del individuo ${ }^{(15)}$. Esta crítica se acompañó, además, de una extensa revisión bibliográfica realizada por Fernando Cabieses, becario del Instituto de Biología Andina en la Universidad de Pensilvania, sobre la acción antifatigante de la coca ${ }^{(16)}$.

La respuesta de Gutiérrez Noriega fue muy dura, y se tradujo en una serie de trabajos publicados por él y sus colaboradores. En particular, destacan dos artículos de respuesta, uno publicado en Anales de la Facultad, titulado "El cocaísmo y la alimentación en el Perú", donde luego de una erudita revisión de todos los testimonios históricos, deja muy clara la relación entre consumo de hojas de coca y trabajo forzado, para luego analizar las penosas condiciones de alimentación, expresadas en de calorías per cápita en los departamentos de mayor consumo de coca, donde, además, estaba asociada a una alta prevalencia de consumo de alcohol (17). El otro ensayo, titulado "Errores de interpretación del cocainismo en las grandes alturas", independientemente de la dureza y los juicios de valor, proporcionales a los recibidos en el ensayo de Monge, Gutiérrez Noriega despliega uno de los más inspirados alegatos en la historia del positivismo peruano, desarrollando con una rigurosa lógica, todo un conjunto de evidencia desde una amplia gama de disciplinas, desde la historia hasta la farmacología experimental, para desmontar una por una, las tesis de Monge. Termina señalando lo inadmisible del concepto de raza climatofisiológica dentro de la biología y de la antropología, para concluir, con cierta ironía, de que sería muy loable que, como consecuencia de los estudios de fisiología de altura, se diera una legislación más apropiada para el trabajo en las grandes alturas y se mejorara las condiciones de higiene y la pésima alimentación de los habitantes de los andes ${ }^{(18)}$.

A pesar de la polarización entre quienes encarnaban la tradición de la Escuela Médica Peruana y quienes la cuestionaban desde un positivismo alternativo, estaban quienes, desde la medicina social, cuestionaban el consumo de coca, pero que se posicionaban de una manera distinta, frente al problema indígena, un ejemplo de ello fue Max Kuczynski, quien en su obra escrita con Carlos Enrique Paz Soldán "Disección del indigenismo peruano" deslindó con ambas posiciones en disputa. En ese sentido, rechazó entrar en cuestiones fisiológicas u toxicológicas, ni de experiencias de laboratorio, todas ellas condiciones que simplifican la realidad, optando por la observación del cocainismo como realidad social y, por lo tanto, de la problemática de determinados grupos afectados por una exclusión severa, asociada por un modelo de economía agraria, basada en haciendas, cuya alta rentabilidad se obtenía a partir de indígenas en situación de siervos de la gleba, explotados en condiciones de cocainismo y alcoholismo severo, descritos como "valles de coca y de aguardiente" (19). Desde, esa perspectiva, para Kuczynski, el problema debía ser solucionado convirtiendo a las poblaciones indígenas en ciudadanos, integrándolos a un piso de protección social. En el punto más álgido del debate, Max Kuczynski estaba diseñando un modelo de operación para llevar salud a las poblaciones indígenas, de mayor alcance y densidad de servicios e intervenciones sanitarias adaptadas a la complicada geografía y a las necesidades sociales de dichas poblaciones ${ }^{(20)}$.

Los acontecimientos tendían a complicar esta controversia. Ante la dureza del debate interno, el Gobierno del Perú solicita en abril de 1947, a la Comisión de Estupefacientes de las Naciones Unidas, el envío de expertos para dilucidar los efectos del consumo de las hojas de coca en las poblaciones, en un contexto de enrarecimiento de la situación política del país. La insurrección aprista en El Callao genera una crisis que cambia dramáticamente la correlación de fuerzas en la Universidad de San Marcos, con la huida del rector, Luis Alberto Sánchez, destacado líder del APRA, al ser declarado este partido fuera de la ley ${ }^{(21)}$. El decano de la Facultad de Medicina, Sergio Bernales, principal apoyo de Gutiérrez Noriega en la universidad, fue destituido por el Consejo de Facultad en un movimiento liderado, entre otros, por Alberto Hurtado, ministro de Salud en ejercicio e investigador principal del Instituto de Biología Andina ${ }^{(22)}$. El 27 de octubre del mismo año, se dio el golpe militar de Manuel A. Odría, que terminaría de configurar una redistribución de todas las esferas del poder. Ese cambio de correlación de fuerzas terminó favoreciendo a Monge, y le permitió tener una fuerte llegada a nivel político, pero no bajó la intensidad del debate, el cual fue acompañado de sutiles y, a veces, explícitos gestos de hostilidad por ambas partes. Pronto, ambos puntos de vista aparecieron publicados en la revista América Indígena ${ }^{(23)}$, donde Gutiérrez Noriega aprovechó para protestar por el cierre de su laboratorio en el Instituto Nacional de Higiene, además de señalar que: El castigo y la persecución recaen, en este caso, sobre quienes se atreven a señalar los graves efectos tóxicos de la droga (coca) (24)

En marzo de 1949 se constituyó la Comisión de Expertos para el Estudio sobre los efectos de la Hoja de Coca, quienes, pocos meses después, llegaron al Perú. Como era de esperarse, sus actividades constituyeron un nuevo escenario de confrontación. Durante tres meses, dicho grupo de trabajo pudo revisar toda la evidencia disponible y tomar pulso a los niveles de la controversia existente en el país, de hecho, el informe de la comisión es un extraordinario registro del debate 
público en torno a las condiciones de la población indígena peruana. Si bien el Gobierno de Odría nombra a Carlos Monge presidente de una comisión que se va a constituir como contraparte al equipo de expertos de las Naciones Unidas, tuvo poco éxito en lograr algún nivel de incidencia. Su informe final, publicado en julio de 1950, da cuenta del hecho que los enfoques alternativos al pensamiento de Monge ofrecían mejores marcos interpretativos para explicar la situación de las poblaciones andinas peruanas. Señalan que: Nadie ha descrito como Kuczynski las miserables condiciones higiénicas y sociales en que viven las poblaciones indígenas. Respecto a la evidencia sobre la acción de la hoja de coca, la comisión en varios pasajes de su informe señala la importancia de las observaciones de Gutiérrez Noriega y de su equipo de colaboradores, las cuales constituyeron el núcleo de evidencias en torno a sus conclusiones sobre la acción de la coca. Fue un duro golpe para Monge el hecho de que la comisión, señale explícitamente que no es necesario considerar a los indígenas andinos como una raza en la cual existen leyes fisiológicas especiales ${ }^{(25)}$. Como consecuencia de ello, Monge despliega una argumentación orientada a sostener que, frente a la controversia, el informe de la comisión de expertos constituye una tercera opinión y que en realidad se requerían mayores investigaciones sobre el hábito del consumo de hojas de coca ${ }^{(26)}$.

En junio de 1951 Carlos Monge fue nombrado director del Instituto Indigenista Peruano y, posteriormente, codirector del proyecto Perú-Cornell -un convenio entre el departamento de Sociología de la Universidad de Cornell y el Instituto Indigenista Peruano, cuyos objetivos eran, a partir del desarrollo de investigaciones en comunidades andinas, construir: Un sistema social y económico más productivo, y contribuir al desarrollo de gente más capacitada y segura de sí misma (27). En términos explícitos, el proyecto iniciaba de la decisión política de modernización de las poblaciones indígenas a partir de diversas intervenciones de carácter desarrollista y civilizatorio (28). En ese escenario, el discurso de Monge estaba más orientado a impulsar los programas de aculturación en la esfera del Instituto Indigenista, y trató, en esta última etapa, de expandir sus concepciones de fisiología antropológica hacia las relaciones de equilibrio con el medioambiente ${ }^{(29)}$.

\section{DISCUSIÓN}

El debate sobre la aclimatación a la altura y el hombre andino, probablemente fue la primera gran controversia en la historia del positivismo peruano, donde frente a un paradigma y a un colectivo investigativo, emerge una visión opuesta del hombre andino. Carlos Monge, probablemente muy influenciado por el indigenismo de los años $\mathrm{XX}$, construye una imagen progresista del indígena peruano a través de sus excepcionales condiciones de adaptación a la altura y sobre esta piedra angular obtiene un enorme logro investigativo, el descubrimiento de la enfermedad de los Andes. Esto le permite, en un país con enormes dificultades, conseguir recursos, articular una incipiente comunidad científica construir un piso de institucionalidad y lograr reconocimiento internacional, en el lapso de una década, un logro sin precedentes en la historia de la ciencia peruana del siglo XX, uno de los ejemplos más notables de desarrollo de la ciencia desde la adversidad ${ }^{(30)}$. Sin embargo, desde diversas perspectivas se fueron desplegando visiones alternativas que no tuvieron posibilidades de dialogar con el paradigma dominante. Todo se tradujo en dos imágenes opuestas, un indio en condiciones de explotación minera - agrícola subyugado por la coca constituida como una toxicomanía, desde la mirada de Gutiérrez-Noriega, que recoge la mirada del activismo del APRA auroral en los trabajadores de las haciendas del norte ${ }^{(31)}$, opuesto a la visión de un indígena andino en proceso de adaptación permanente a la agresión climática, que necesita la hoja de coca era necesaria para desempeñar las actividades diarias y sobrevivir en medios tan hostiles y con una calidad de vida marcada por la pobreza, desde el paradigma investigativo de Monge ${ }^{(32)}$.

Desde el lado de Monge, este estaba absolutamente convencido de su posición, ello se observa claramente en una entrevista, donde señala su perspectiva de cómo debe abordarse el problema indígena: Como todo problema, el indígena debe tener una solución... los aportes son cuantiosos, pero se frustraron por la unilateralidad del empeño, que no tomo en consideración la complejidad del asunto y la necesidad de integrar, por consiguiente, todas las variables del conocimiento. Se trabajó más desde el gabinete y menos desde el hombre mismo, motivo del problema. En un determinado momento de la entrevista, señala la tradicional falta de apoyo a la investigación: Lo que caracteriza la investigación en el Perú es el sacrificio de los que buscan la verdad... pero eso no basta para la obra de aplicación científica en el hombre y el campo. Por eso es de esperar que el financiamiento de los proyectos de aculturación al servicio del hombre andino, que el Instituto Indigenista Peruano contempla, reciba el financiamiento necesario... (33). En este sentimiento aparecen todos los ingredientes del escenario de fragmentación del tejido social investigativo peruano a mitad del siglo XX: una incapacidad de escuchar al otro, a partir de rígidas apuestas paradigmáticas en un escenario de precariedad institucional y de escasez, donde la cercanía al poder era fundamental para garantizar el acceso a recursos y legitimidad política y social, terminó por consolidar una dinámica, donde la hegemonía de un colectivo de pensamiento determinó la desaparición de otras corrientes investigativas del escenario académico y social. 
En un artículo de 1957, denominado "Antropología y medicina", Carlos Monge intenta escribir la memoria y el canon de la antropología médica peruana, excluyendo explícitamente a Carlos Gutiérrez Noriega y a Max Kuczynski ${ }^{(34)}$. Ello refleja la forma en que terminó este debate, con un empate catastrófico, donde no solamente perdieron todos, sino, fundamentalmente, perdió el Perú. Para el año 1949, era claro en la discusión del nuevo estatuto universitario de San Marcos, que la visión durante la gestión de Bernales de una Facultad de Medicina con tres institutos de investigación (Altura y Biología Andina, Medicina Social y Farmacología Experimental) era parte del pasado. En 1950, Kuczynski estaba ya desvinculado de la Facultad de Medicina y los programas de inclusión sanitaria orientados a las poblaciones indígenas, fruto del esforzado trabajo de una década, habían sido desactivados. Asimismo, las investigaciones sobre cocaína habian sido paralizadas, dado el cierre del laboratorio de Farmacología Experimental del Instituto de Higiene y Salud Pública, a lo que siguió la temprana desaparición de Gutiérrez Noriega en un accidente automovilístico en Europa en 1950. Toda la vorágine investigativa en torno al hábito de la coca y a su contexto médico-social desapareció, en un contexto de hegemonía de las líneas investigativas de Hurtado, sin mayor punto de encuentro con el mundo social y del escaso margen de maniobra que tuvo Monge como director del Instituto Indigenista y codirector del Proyecto Perú-Cornell, además del poco éxito que tuvo para acceder a recursos que le permitieran desplegar nuevas investigaciones en torno a sus ideas (28). Finalmente, fueron decisiones a nivel de la esfera de la política internacional, en torno a la erradicación de la hoja de coca ${ }^{(35)}$ y un gobierno autoritario como el de Odría, que entendía las políticas hacia las poblaciones indígenas en términos civilizatorios ${ }^{(36)}$, las que dieron por cerrada; una de las mayores controversias científicas de la historia investigativa peruana del siglo XX.

¿Qué nos deja todo este recuento de encarnizados debates, de profundos desgarramientos institucionales y oportunidades perdidas? Reflexionando desde el ámbito de la salud pública, considero que nos muestra el germen de una cultura de fragmentación y desacumulación profundamente enraizada en nuestra comunidad académica y en nuestras instituciones en general (37), una enorme incapacidad para escuchar al otro, para establecer puentes entre distintas posiciones, una falta de vocación para la acción colectiva, asociada a una cultura autoritaria y a lo que Marcos Cueto denominó como esa vocación para volver a empezar ${ }^{(38)}$, lo que nos conduce, finalmente, a una incapacidad para poder generar consensos respecto a grandes políticas públicas, lo que nos deja con una cada vez menor capacidad de decisión respecto a un escenario de políticas globales, en un mundo cada vez más interdependiente, donde nuestra fragmentación nos hace vulnerables a la introducción de modelos y soluciones que vienen de otros centros de decisión y que no recogen las verdaderas necesidades y expectativas de la sociedad peruana. Si algo podemos rescatar de este debate, es la necesidad de imaginar una forma alternativa de diálogo y escucha que nos pueda constituir en una comunidad académica nacional, paso necesario, para iniciar, como decía el recordado filósofo sanmarquino Juan Abugattas, ese enorme esfuerzo de sueños y voluntades para construir en el Perú, esa modernidad republicana, indispensable para enfrentar los enormes retos que nos plantea el siglo XXI.

Fuentes de financiamiento: autofinanciado.

Conflictos de interés: el autor declara no tener conflictos de interés.

\section{REFERENCIAS BIBLIOGRÁFICAS}

1. Cueto M. Excelencia Científica en la periferia. Actividades Científicas e Investigación Biomédica en el Perú 1890-1950. Lima: Grade-Concytec; 1989.

2. Lossio J. El peruano y su entorno. Aclimatándose a las alturas andinas. Lima: Instituto de Estudios Peruanos; 2012.

3. Fleck L. La génesis y el desarrollo de un hecho científico. Introducción a la teoría del estilo de pensamiento y del colectivo de pensamiento. Madrid: Alianza Editorial; 1986.

4. Monge Medrano C. La Enfermedad de los Andes. An Fac med.1928;11(12):90-1.
5. Monge Medrano C. Unanue y la Cosmometeoropatología. Discurso Académico de Apertura a la Asamblea Médica Conmemorativa del Centenario de Unanue. Lima: Lux de E. L. Castro; 1933.

6. Monge Medrano C. Actas de la Segunda Conferencia Panamericana de Eugenesia y Homicultura de las Repúblicas Americanas. Buenos Aires: 1934. Frascoli y Bindi; 78-81 p.

7. Monge Medrano C. Política Sanitaria Indiana y Colonial en el Tahuantinsuyo. An Fac med. 1935;17(2):233-76.

8. Monge Medrano C. La vida en los Andes y el Mal de Montaña Crónico. An Fac med. 1942, 25(1):1-18.
9. Información Médica. La colectividad médica rinde homenaje al profesor Monge. Actual Med Peru. 1941;7(8):248-54.

10. El Indigenismo desde el punto de vista médico y antropológico. [Editorial]. Actual Med Peru, 1936;1(11):617-18.

11. Gutiérrez-Noriega C. Biotipología del Perú Antiguo. Actual Med Peru. 1936; 2(3):118-30.

12. Gutiérrez-Noriega C. Diseño de un Psicológico y Antropológico de la Raza Amerindia. Actual Med Peru, 1937;3(4):159-95.

13. Gutiérrez-Noriega C. Estudios sobre la Coca y la Cocaína. Lima: Ministerio de Educación Pública; 1947. 
14. Monge Medrano C. Biología Andina y de Altitud. Aclimatación y Mal de Montaña Crónico en las altiplanicies del Perú, México y Estados Unidos, sus relaciones con la Medicina de Aviación. Lima:Informe a la Facultad de Medicina; 1947.

15. Monge Medrano C. El Problema de la Coca en el Perú. An Fac med. 1946;39(4):311-15.

16. Cabieses MF. La acción antifatigante de la cocaína y la habituación de la Cocaína y la habituación a la Coca en el Perú. An Fac med. 1946;39(4):316-66.

17. Gutiérrez-Noriega C. El Cocaísmo y la alimentación en el Perú. An Fac med. 1948,31(1):1-90.

18. Gutiérrez-Noriega C. Errores sobre la Interpretación del Cocaísmo en las Grandes Alturas. Rev Farmacol Med Exp. 1948;1(1):100-23.

19. Kuczynski M, Paz Soldán CE. Disección del Indigenismo Peruano. Lima: Publicaciones del Instituto de Medicina Social, 1948, 86-93 p.

20. Kuczynski M. El Cuzco, futuro centro de la reorganización socio-económicosanitaria del sur. La Reforma Médica; 1945. 623-33.

21. Ortiz SJ. Las Rebeliones Navales del Callao 1932 y 1948. Apuntes. 1999;45:83-95.

22. Paz Soldán CE. El Colapso de la Facultad de Medicina. La Reforma Médica. 1948, XXXIV (532-533):290-6.

23. Monge MC. Aclimatación en los Andes. América Indígena, 1949, 9(4):267-286.

24. Gutiérrez-Noriega C. El hábito de la Coca en el Perú. América Indígena. 1949;9(2):143-54.
25. Naciones Unidas. Informe de la Comisión de Estudio de las Hojas de Coca. Nueva York: Consejo Económico y Social; 1950.

26. Monge Medrano C. La Necesidad de estudiar el problema de la masticación de las hojas de coca. América Indígena. 1953;13(1):47-54.

27. Holmberg AR. Vicos: Método y Práctica de Antropología Aplicada. Lima: Editorial Estudios Andinos; 1966. $25 \mathrm{p}$.

28. Gonzáles O. El Instituto Indigenista Peruano: Una historia por conocer. En: Giraudo L, Martín-Sánchez J, Editores. La ambivalente historia del indigenismo. Campo interamericano y trayectorias nacionales 1940-1970. 1ra Ed. Lima: Instituto de Estudios Peruanos; 2011. 146-51 p.

29. Monge Medrano C. La vida en las Altiplanicies Andinas. Procesos Ecológicos. Perú Indígena, 1963;10(24-25):9-21.

30. Cueto M. La Ciencia de la adversidad. Un esbozo de la historia de la Ciencia en el Perú. Unodiverso: Ciencia, Tecnología y Sociedad. 2005; (1):2239.

31. Klaren P. Formación de las haciendas azucareras y los orígenes del APRA. 2da Ed. Lima: Instituto de Estudios Peruanos; $1976.75-81 \mathrm{p}$.

32. Meza Bazan M. El enfoque médico social sobre el uso y consumo de la coca y la cocaína en el Perú en la primera mitad del siglo XX. Nueva Coronica. 2013;(2):487-503.

33. Monge Medrano C. Entrevista Periodística: El Problema Indígena y el Proyecto Perú-Cornell. 1951.
Documento E.09.085. Archivo Personal de Carlos Monge. Fondo Documental de la Biblioteca de la Pontificia Universidad Católica del Perú.

34. Monge Medrano C, Vazquez M. Antropología y Medicina. Perú Indígena. 1957;6(14-15):19-33.

35. Gootenberg P. Cocaína Andina. El proceso de una droga global. Puno: Universidad Nacional de Juliaca; 2016. 335-43 p.

36. Murillo JP, Salaverry GO, Franco PG. País Enfermo y Despoblado. Debates Sanitarios y Proyectos de Desarrollo en el Perú en la primera mitad del siglo XX. En: Hochman G, Di Liscia MS, Palmer S .Patologías de la Patria: Enfermedades, Enfermos y Nación en América Latina. 1ra Ed. Buenos Aires: Lugar Editorial; 2012, 155-180 p.

37. Murillo JP, Mendoza W, Franco PG. ¿Quo Vadis Salud Pública? Discurso y práctica de la Salud Pública en el Perú y su Auge Universitario en la Década de los Noventa?. An Fac med. 2000; 61(2):146-60.

38. Cueto M. La Vocación para volver a empezar: Las políticas de población en el Perú. Rev Peru Med Exp Salud Publica. 2006,23(2):123-31.

Correspondencia: Juan Pablo Murillo Dirección: Av. San Felipe 647-1502-B, Jesús María. Lima, Perú. Teléfono: (511) 999746449

Correo electrónico:jmurillop@unmsm.edu.pe

\section{Nuestros artículos se encuentran indizados en:}

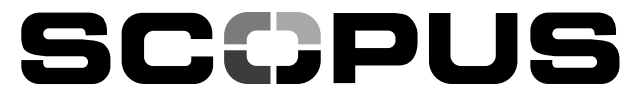

